\title{
Effectiveness of teamwork and communication education using an interprofessional high- fidelity human patient simulation critical care code
}

\author{
Deborah D. Garbee ${ }^{1}$, J ohn T. Paige ${ }^{2}$, Laura S. Bonanno ${ }^{1}$, Vadym V. Rusnak ${ }^{3}$, Kendra M. Barrier ${ }^{1}$, \\ Lyubov S. Kozmenko ${ }^{1}$, Quingzhao $\mathrm{Yu}^{4}$, J ean E. Cefalu ${ }^{1}$, T. Kirk Nelson ${ }^{3}$ \\ 1. School of Nursing, Louisiana State University, USA. 2. School of Medicine, Louisiana State University, USA. 3. School of \\ Allied Health, Louisiana State University, USA. 4. School of Public Health, Louisiana State University, USA.
}

Correspondence: Deborah D. Garbee. Address: 1900 Gravier Street, New Orleans, LA 70112. Telephone: 504-568-4183. Fax: 504-568-5853. Email: dgarbe@Isuhsc.edu.

Received: June 1, 2012

DOI : 10.5430/jnep.v3n3p1
Accepted: July 5, 2012

URL: http://dx.doi.org/10.5430/jnep.v3n3p1

\section{Abstract}

Background: Effective teamwork and communication among healthcare professionals are essential to ensure quality patient care and safety. The use of high-fidelity human patient simulation scenarios enhances learning and has been used in various health professions education. The purpose of this study was to evaluate the efficacy and retention of teaching team-based competencies to interprofessional student teams using high-fidelity simulation.

Methods: Quasi-experimental, pre/post-test design was used. The study was a teaching intervention using high-fidelity human patient simulation with crisis resource management techniques to teach team based competencies. A convenience sample of students from medicine, nurse anesthesia, undergraduate nursing, and physical therapy participated. There were pre/post data collected for fall and spring simulations from both participants and observers. Key variables were the various teamwork competencies. Participants and trained observers rated teamwork behaviors, each using two measures. Mean scores on participant and observer rated tools were compared using paired samples $t$-tests.

Results: A total of 35 students underwent training in the fall of 2009 and 25 of the students returned in the spring of 2010 for repeat training. Participant paired samples $t$-tests showed a significant increase from simulation scenario one to two ( $p$ $<.05$ ) in both the fall and spring. Observer data showed significant improvements in mean scores on one measure.

Conclusions: High-fidelity simulation appears to improve both perceived and actual team-based competencies with retention over time. Findings support the benefit to students of repeat training in a six month period and provide some validation for faculty time and effort in repeated simulations.

\section{Key words}

Interprofessional education, Human patient simulation, Medical students, Nursing students, Physical therapy students 


\section{Introduction}

Effective teamwork and communication among healthcare professionals is essential to ensure quality patient care and safety. Poor communication and teamwork among healthcare providers can lead to adverse patient events, higher patient complication rates, medical errors and increased patient mortality ${ }^{[1,2]}$. The use of team training among interprofessionals is an effective methodology when utilized to improve patient safety and reduce medical error, especially since teams make fewer mistakes when every member of a team is aware of each teammate's responsibilities ${ }^{[3]}$. The Institute of Medicine (IOM) has established the ability to work in interdisciplinary teams as one of its core competencies for healthcare professionals and recommends that all health care team members have a clear understanding of each other's roles and responsibilities ${ }^{[4]}$. This study explored the effectiveness of bringing together teams of four health care professions students for high-fidelity simulation team training.

\section{Literature review}

Healthcare professional education curricula rarely include interdisciplinary or collaborative educational experiences;

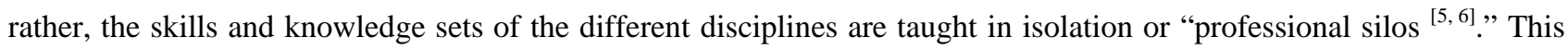
"silo mentality" fosters competition and hierarchy instead of teamwork and collaboration and can contribute to a dysfunctional healthcare team ${ }^{[5,7]}$. National agencies including the IOM, the Joint Commission, the Agency for Healthcare Research and Quality (AHRQ), the Pew Commission, and the Carnegie Foundation have emphasized the importance of interprofessional education, specifically in the areas of teamwork and communication, to instill positive teamwork behaviors early in the academic preparation of healthcare professionals ${ }^{[4,5,8,9]}$. In addition, the IOM Health Professions Education: A Bridge to Quality ${ }^{[4]}$ proposes the establishment of an interdisciplinary health education organization to identify and evaluate models of education that promote best practices in healthcare. Professional socialization begins during the educational process as attitudes toward collaboration develop as students are socialized into their discipline ${ }^{[10]}$.

The use of interprofessional simulation based training (SBT) for healthcare professionals is supported in the literature. The Lucien Leape Institute, within the National Patient Safety Foundation, promotes the utilization of simulation-based training for inter-professional education of healthcare professionals to provide students the skills necessary to work effectively in teams ${ }^{[11]}$. High fidelity simulation-based training (HFSBT) is effective in team-oriented training because it provides the opportunity for teams to work together in treating low frequency, high risk events in an immersive and safe learning environment, with the ability to learn from "mistakes" without consequences to patients ${ }^{[12]}$.

Dillon and colleagues ${ }^{[10]}$ used a pre/posttest design to assess nursing and medical students' perceptions of interdisciplinary collaboration with a mock code experience. Although limited by convenience sample and small sample size, the findings of this study suggest that early exposure of students to interdisciplinary collaboration as part of the educational process may establish a foundation for collaboration that continues through their professional practice ${ }^{[10]}$. Capella and colleagues ${ }^{[13]}$ reported that structured trauma resuscitation team training augmented by simulation improves team performance resulting in improved efficiency of patient care during a trauma. Meanwhile, Siassakos and colleagues $^{[14,15]}$ reported on utilization of SBT to in-service professional teams and reported that there were no significant correlations between outcomes and individual team members' knowledge, skills, or attitudes. However, they reported that the teams with the best outcomes showed significantly better communication skills, especially in the areas of hand-off communication using the Situation, Background, Assessment, and Recommendation (SBAR) format for report and task allocation.

Although several studies suggested that HFSBT of inter-professional student teams does improve students' team-based attitudes ${ }^{[10,16,17]}$, the impact of distributed HFSBT of such student teams on the acquisition and retention of team-based skills and attitudes is not known. Therefore, the purpose of this study was to evaluate the efficacy and retention of teaching team-based competencies to interprofessional student teams using high-fidelity simulation. The following hypotheses 
were considered for this study: (1) participants in high fidelity SBT will demonstrate increased teamwork and communication skills after each training session as measured by both direct observation and participant self-rating, and (2) participants in high fidelity SBT will demonstrate retention of teamwork and communication skills after six months.

\section{Methods}

\subsection{Design}

A quasi-experimental, pre/post-test design was used. Training was distributed over an approximately six month period. Initial high-fidelity SBT occurred in fall, 2009. Participants were then invited back to undergo a second training session in spring, 2010. This second session was structured in the same manner as the first session and included the same scenarios.

Expedited Institutional Review Board (IRB) approval with waiver of documentation of informed consent was obtained prior to initiation of the study protocol.

\subsection{Setting}

Training took place at a Health Sciences Center (HSC) in the southern United States during fall, 2009 and spring, 2010. Interprofessional student teams were recruited from the school of medicine (SOM), school of nursing (SON), and school of allied health (SOAH). The School of Medicine (SOM) enrolls approximately 200 students each year as part of its four year program. The School of Nursing (SON) has approximately 600 students in its undergraduate baccalaureate program, offering a three year traditional and two year accelerated programs. Within the SON, a nurse anesthesia program enrolls approximately 40-45 students per year. Finally, the School of Allied Health (SOAH), Department of Physical Therapy enrolls approximately 40 students a year within a three year doctor of physical therapy (DPT) program.

Sessions took place at a state of the art simulation center that houses several spaces dedicated to high fidelity simulation (HFS), including an emergency/intensive care room. For the training, this space was outfitted as an intensive care unit (ICU) room containing ventilator equipment, a fully equipped code cart, two medical gurneys, airway equipment, and intravenous access (IV) equipment. Activity within the room was video recorded.

Simulation equipment used for the training sessions included a full-scale computer operated human patient simulator (HPS) mannequin (Medical Education Technologies Incorporated [METI], Sarasota, FL). The HPS is capable of producing a palpable pulse as well as breath and heart sounds via an air compressor. In addition, through its software, it is able to mimic human physiologic responses to pharmacologic (e.g., IV administration of epinephrine) and pathologic (e.g., massive hemorrhage, tension pneumothorax) input. For the training, the HPS software had a previously described enhanced user-friendly interface developed at the HSC that facilitated more rapid, complex, and authentic physiologic responses from the simulator, increasing the psychological fidelity (e.g. the suspension of disbelief by the participants) of the simulated scenario ${ }^{[18]}$. This interface permitted the creation of algorithm-designed physiologic responses by the HPS based on participant/team decision making ${ }^{[18,19]}$.

\subsection{Participants}

Participants for the study were a convenience sample $(n=35)$ drawn from senior level medical, nursing, nurse anesthesia, and physical therapy students. Fourth year medical students enrolled in an intensive care (e.g., trauma intensive care or medical intensive care) or emergency room elective agreed to participate in the initial SBT sessions. Undergraduate baccalaureate nursing students enrolled in a senior level critical care course were recruited for participation. Senior level nurse anesthesia students participated in the project. Finally, senior level physical therapy students volunteered for the SBT. 


\subsection{Procedures}

Each student team consisted of two medical students, two undergraduate nursing students, two nurse anesthesia students, and one to two physical therapy students. Fourth year medical students filled the roles of intensivists (e.g. critical care physicians) working in the ICU. Nurse anesthesia students participated as nurse anesthetists rounding in the ICU on pre-operative patients. Senior nursing students assumed the role of intensive care nurses working in the ICU. Lastly, senior physical therapy students acted as physical therapists assigned to the ICU to care for patients. For the first scenario, one student from each profession was designated as the lead individual. For the second scenario, this lead role was switched to the other individual in that profession. All students participated in the debriefing following each scenario. All students were invited back to participate in a second SBT approximately six months later in spring, 2010 with 25 of the original 35 students returning in the spring.

For each training session, two standardized, authentic scenarios were used. Each scenario involved a bedside event designed to catalyze team interaction and promote team-based competencies. The first scenario involved an ICU patient with a cardiac history that was awaiting transfer to the floor and developed unstable atrial fibrillation with rapid response (e.g. a rapid, irregular heart rate associated with low blood pressure) following transfer by PT out of bed for therapy. The second scenario involved the development of a tension pneumothorax (e.g. air around the lung under pressure causing decrease in blood flow to the heart) in an ICU patient awaiting transfer to the floor with a history of lung problems.

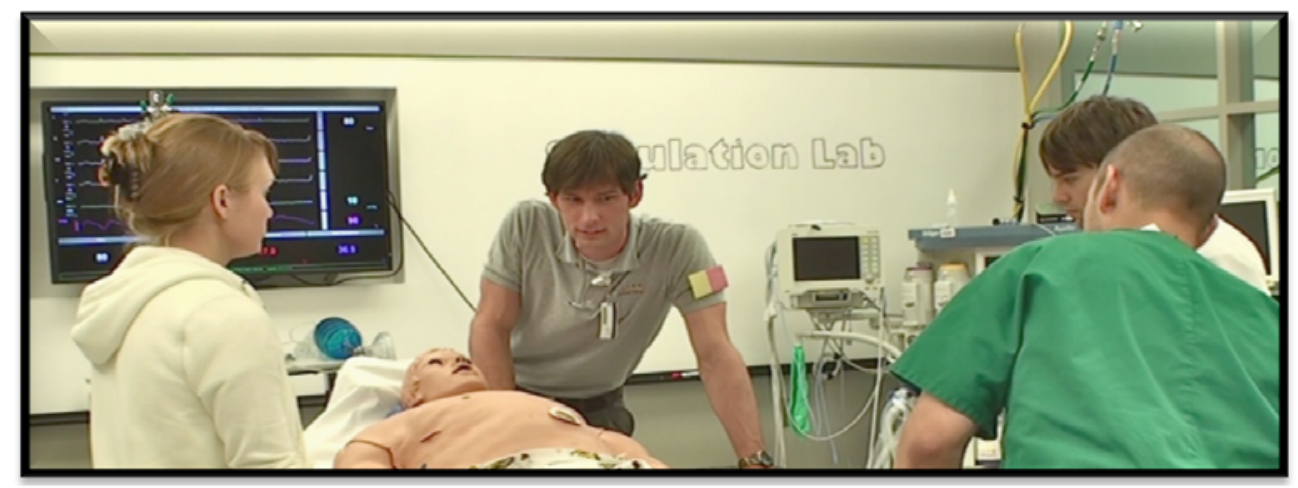

Figure 1. Nursing and Physical Therapy Students Initiate Scenario

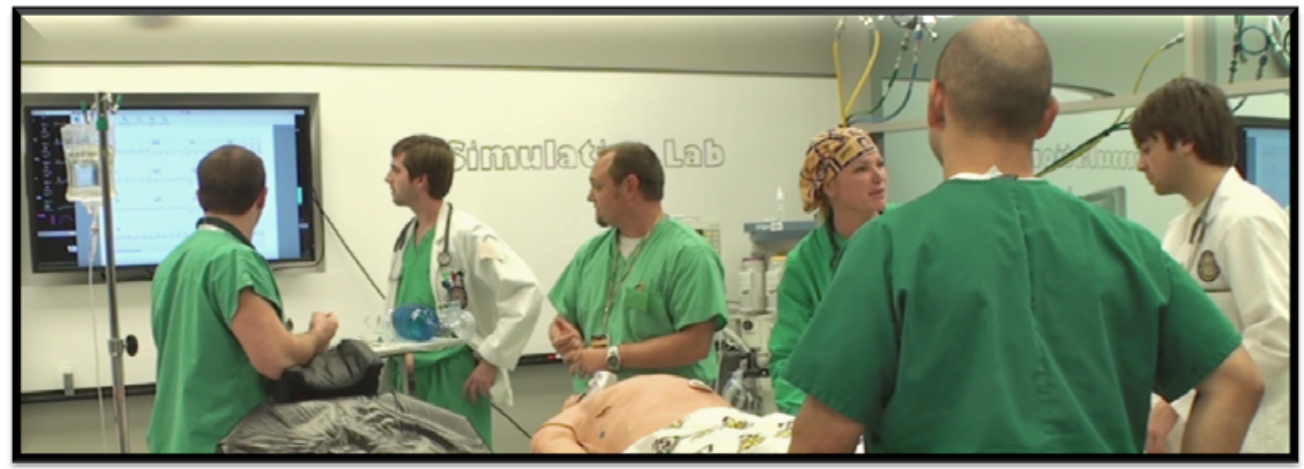

Figure 2. Teamwork during Crisis in Scenario

Each training session followed a previously used dual scenario format (e.g., first scenario, debriefing, second scenario, debriefing) ${ }^{[20,21]}$ and lasted approximately two hours. A brief orientation was conducted prior to each session in which the training goals and objectives, simulation equipment, and training ground rules were reviewed. To assure the psychological safety of the participants, maintaining the confidentiality of the proceedings was emphasized. Upon receiving a brief 
verbal history regarding the "patient," the team would then initiate the scenario (see Figure 1). During the scenario, a crisis arose that participants must manage as a team (see Figure 2). Immediately after scenario completion, all students participated in a facilitator-led, structured debriefing held within the virtual intensive care unit (ICU) space. This focused debriefing encouraged student engagement through reflective practice and emphasized nine key team-based competencies (e.g. shared mental model, resource management, situation awareness, cross monitoring, anticipatory response, open communication, flatten hierarchy, mental rehearsal, and role clarity). The debriefing structure followed a previously described format developed for in situ (e.g. at the point of care in the actual clinical environment) operating room (OR) team training ${ }^{[22,23]}$. In addition to an introduction and closure, key elements of the debriefing included engagement, focus, reflection and critique, and application to everyday practice ${ }^{[18]}$. Within this overall structure, the facilitator drew on several other established formats to maximize learning, including Pearson and Smith's three questions ${ }^{[24]}$, the plus/delta technique ${ }^{[25]}$, and Thiagi's six phases of debriefing ${ }^{[26]}$. In particular, emphasis was made on "what is right," not "who is right" ${ }^{[27]}$. Each training session ended with an overview of the team-based competencies and a student-led discussion on how best to transfer team-based skills learned in the HFS to the actual clinical environment.

\subsection{Outcome measures}

Observer- and participant-based evaluations of team-based performance were conducted after each scenario for every session. For each type of evaluation, two separate rating tools were employed in order to demonstrate convergence of findings with the use of different instruments.

\section{Observer-based evaluation}

For the observer-based evaluation of team-based performance, the two instruments used were the Teamwork Assessment Scales (TAS), a modified version of the Operating Room Teamwork Assessment Scales (ORTAS) ${ }^{[28-30]}$ and the Communication and Teamwork Skills (CATS) ${ }^{[31]}$ assessment tool.

The TAS included two scales: (1) a 5 item individual performance scale focusing on team-based behaviors (TBB) of the individual using a multi-source (e.g. 360 degree) evaluation format and (2) a six item overall teamwork scale evaluating total team performance ${ }^{[29,30]}$. The overall teamwork scale contained two subscales: (1) a three item shared mental model (SMM) subscale and (2) a three item adaptive communication and response (ACR) subscale. Each item contained behavioral anchors for a six point Likert-type scale $(1=$ Definitely No to $6=$ Definitely Yes $)$. Given the multi-source format of the TBB scale, observer ratings of each individual on the ICU team (e.g. medical student, nursing student, physical therapy student, and nurse anesthesia student) were possible.

According to Frankel and colleagues ${ }^{[31]}$, the CATS assessment tool is a behavior-based instrument designed to assess team-based performance in health care regardless of specialty. It contains four categories of 21 specific observable behaviors identified in specialty-specific team rating scale as well as instruments used in aviation. These categories include the following: (1) situation awareness, (2) coordination, (3) communication, and (4) cooperation. This tool is a checklist-based instrument using behavioral anchors for each item and employing a weighted, three-point check system: (1) Observed and Good, (2) Variation in Quality, and (3) Expected but not Observed. Scoring is based on the total number of observations and the quality of the observed performance. The CATS has been used to assess actual clinical performance of surgical teams in practice.

Observers underwent a two hour training session prior to rating actual team performances in the HFS training sessions. This observer training included a didactic PowerPoint ${ }^{\mathrm{TM}}$ lecture focusing on key principles of human factors and team-based science. Following this lecture, TAS and CATS were reviewed item by item, emphasizing the team-based competencies to which each item corresponded. Finally, video recordings of selected prior HFS team training sessions were viewed and rated by the participants using both TAS and CATS. Ratings then were compared among each instrument, and discrepancies were identified, discussed, and resolved. Video rating were then repeated and discussed in 
an iterative process until adequate consensus was reached among all raters regarding scores. For the actual live observer-based rating of performance, at least two observers rated team-based performance for each instrument.

\section{Participant-based evaluation}

Participants rated self-, peer-, and overall team performance after of each scenario using the TAS and the Mayo High Performance Teamwork Scale (MHPTS) ${ }^{[32]}$. The MHPTS is a 16 item scale for participant-based evaluation of team function. It uses a three tiered scoring system: (1) Never or rarely, (2) Inconsistently, and (3) Consistently. It has been demonstrated to be reliable and valid for participant-based evaluation in HFS. The MHPTS, or modification thereof, has also been used to rate videotaped team performance of both students ${ }^{[16]}$ and healthcare providers ${ }^{[33]}$. Additionally, the TAS was administered to participants to allow multi-source evaluation of individual team-based performance. Participant ratings were also calculated for TBB, SMM and ACR.

\subsection{Statistical analysis}

Mean item and subscale scores for each instrument were calculated for observer and participant ratings. The difference between mean calculated scores after each scenario, pre-post, for each subscale was evaluated using a paired samples $t$-test. In addition, paired samples $t$-tests were used to assess retention using scores from the second scenario in the fall to the first scenario in the spring. Overall gains were determined with paired samples $t$-test on scores from simulation one in the fall to simulation two in the spring.

\section{Results}

Participants for the study were drawn from a convenience sample of fourth year medical students, first semester-senior level undergraduate nursing students, senior level nurse anesthesia students, and senior level physical therapy students. The fall 2009 sessions were conducted with 35 participants. Total participants returning for the spring 2010 sessions were 25 participants. Demographic data are displayed in Table 1. Participants were almost evenly represented by males and females, $51.4 \%$ and $48.6 \%$ respectively in the fall and $56 \%$ and $44 \%$ in the spring. Representation by professional program was more homogenous in the fall, $20 \%-28.6 \%$, compared to the spring simulations where nursing accounted for $40 \%$ and $32 \%$ of participants with medicine totaling $16 \%$ and physical therapy $12 \%$. Caucasian students were the largest group (80\% in fall, $72 \%$ in spring) followed by African American, Asian, and Latino students.

Table 1. Demographic Data

\begin{tabular}{|c|c|c|c|c|}
\hline Characteristics & Fall 2009 & $\%$ & Spring 2010 & $\%$ \\
\hline \multicolumn{5}{|l|}{ Gender } \\
\hline Male & 18 & 51.4 & 14 & 56.0 \\
\hline Female & 17 & 48.6 & 11 & 44.0 \\
\hline \multicolumn{5}{|l|}{ Ethnicity } \\
\hline Caucasian & 28 & 80.0 & 18 & 72.0 \\
\hline African American & 3 & 8.6 & 4 & 16.0 \\
\hline Latino & 1 & 2.9 & 1 & 4.0 \\
\hline Asian & 2 & 5.7 & 2 & 8.0 \\
\hline No Response & 1 & 2.9 & 0 & 0 \\
\hline \multicolumn{5}{|l|}{ Specialty Professional Program } \\
\hline Medical Student & 8 & 22.8 & 4 & 16.0 \\
\hline Undergraduate Nursing Student & 10 & 28.6 & 10 & 40.0 \\
\hline Nurse Anesthetist Student & 10 & 28.6 & 8 & 32.0 \\
\hline Physical Therapy Student & 7 & 20.0 & 3 & 12.0 \\
\hline
\end{tabular}




\section{Hypothesis one}

A paired samples $t$-test was calculated to compare mean participant scores from simulation one to mean scores on simulation two on both the TAS and the MHPTS during fall 2009 and spring 2010 simulations. Comparisons of mean scores from participants on the TAS are displayed in Figure 3. A significant increase from simulation one to simulation two in the fall was found $(p<.05)$ for team-based behaviors (TBB) M=4.75, 5.62, shared mental model (SMM) M= 4.76, 5.62, and adaptive communication and response (ACR) $M=4.74$, 5.58. Spring simulations scores had significant increases as well from simulation one to two, TBB $(M=5.24,5.79)$, SMM $(M=5.22,5.81)$, and ACR $(M=5.11,5.87)$. Mean scores on the MHPTS also showed a significant increase from simulation one to simulation two in both the fall $(\mathrm{M}=21.11$, 25.81) and spring ( $M=21.96,25.76)$ simulations (see Figure 4).

To evaluate observer scores, a paired samples $t$-test was calculated to compare mean observer scores on TAS and CATS from simulation one to simulation two for both fall 2009 and spring 2010 simulations. A significant increase in scores from simulation one to simulation two in fall 2009 was found $(p<.05)$ for TBB $(\mathrm{M}=3.56,4.75)$, SMM $(\mathrm{M}=2.99,4.90)$ and ACR $(M=2.95$, 4.92) (see Figure 5). Although mean scores on the CATS increased in all four subscales from simulation one to simulation two in fall of 2009, only Situational Awareness $(M=86.77,90.75)$, Cooperation $(M=73.9,89.27)$, and Communication ( $M=79.45,96.50)$ scores showed significant increases (see Figure 6).

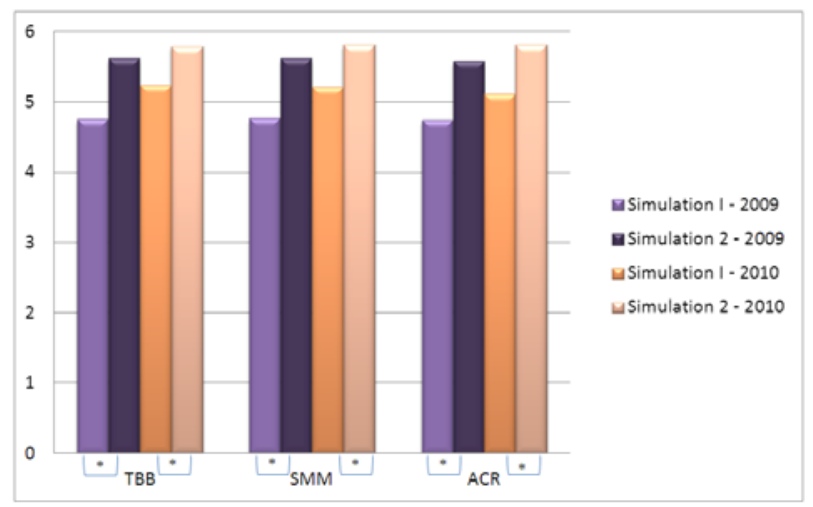

Figure 3. Participant Mean Scores TAS

ACR = Adaptive Communication and Response, SMM = Shared Mental Model, TAS = Teamwork Assessment Scale, TBB = Team-based behaviors, * $p=<.05$, All scores had significant increases from simulation one to two in both fall 2009 and spring 2010.

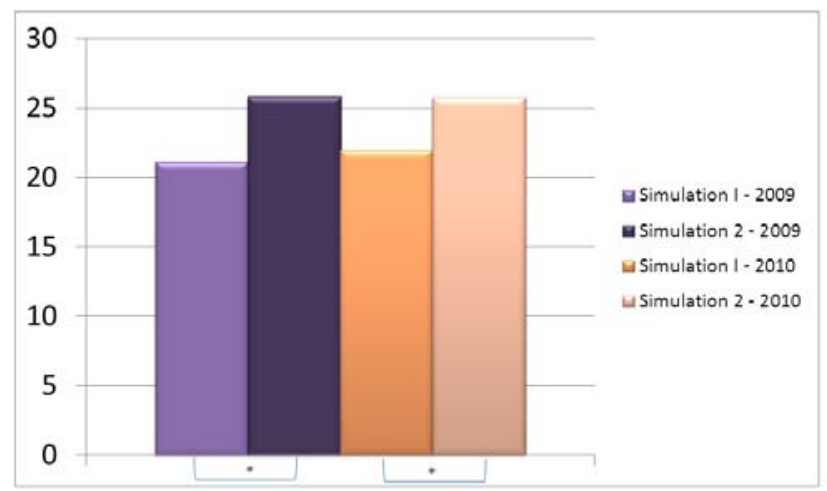

Figure 4. Participant Mean Scores MHPTS

${ }^{*} p=<.05$, All scores had significant increases from simulation one to two in both fall 2009 and spring 2010. 
Similarly, a significant increase in observer mean scores from simulation one to simulation two in spring 2010 was found $(p<.05)$ for TBB $(\mathrm{M}=4.31,4.95)$, SMM $(\mathrm{M}=4.17,5.05)$ and ACR $(\mathrm{M}=4.55,4.95)$ (see Figure 5). Although mean scores on the CATS increased in all subscales, there were not significant increases from simulation one to simulation two in spring 2010 (see Figure 6).

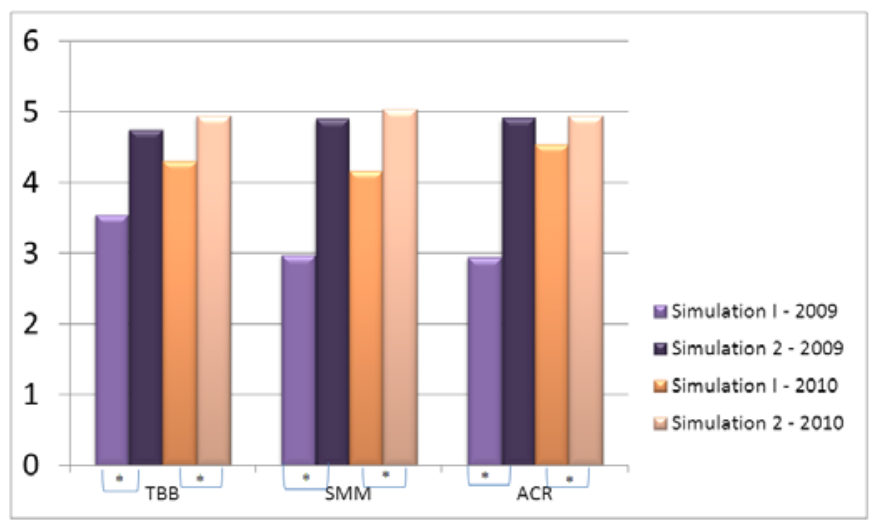

Figure 5. Observer Mean Scores TAS

ACR = Adaptive Communication and Response, $\mathrm{SMM}=$ Shared Mental Model, TAS $=$ Teamwork Assessment Scale, TBB = Team-based behaviors, ${ }^{*} p$ $=<.05$, There were significant increases in TBB, SMM, and ACR from simulation one to two in both fall 2009 and spring 2010.

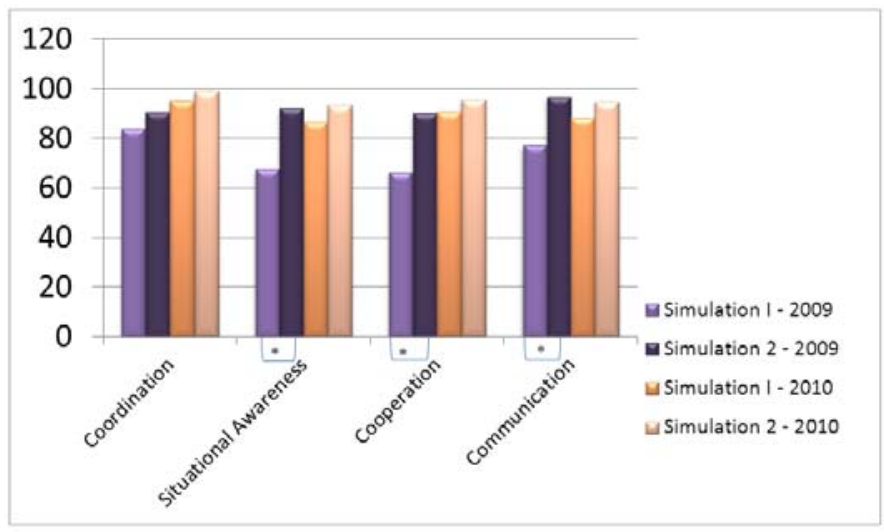

Figure 6. Observer Mean Scores CATS

CATS $=$ Communication and Teamwork Skills, ${ }^{*} p=<.05$, The only significant increase in scores between simulation one and two were in fall 2009-on Situational Awareness, Cooperation, and Communication. Although there were increases in scores in spring 2010 , they were not significant increases.

\section{Hypothesis two}

To assess retention from fall to spring, paired samples $t$-test was calculated on participant mean scores for simulation two in the fall to simulation one in the spring. All mean scores for TBB, SMM, ACR, and MHPTS showed a significant decrease in the spring. Similarly, trained observer scores from simulation two in the fall and simulation one in the spring were analyzed using paired samples $t$-test and a significant decrease in mean scores were found for TBB, SMM, and ACR. However, CATS scores for simulation one in the spring were not significantly different than scores in simulation two in the fall, $p>.05$. 
To assess overall gain from training paired samples $t$-tests were calculated on participant and observer scores for simulation one in the fall to simulation two in the spring. A significant increase in participant scores was found on TBB, SMM, ACR, and MHPTS $(p<.05)$. There were also significant increases in observer scores on TBB, SMM, ACR, and all subscales of CATS $(p<.05)$.

\section{Discussion}

TBB, SMM, and ACR showed significant improvements by both participants and observer ratings. Thus, hypothesis one was supported. Only small losses in skill retention were documented by participant and observer mean scores from the fall to spring simulation on TAS and MHPTS, with improvement in communication, cooperation, coordination, and situational awareness. Hypothesis two was not supported. Results of this study support the work of others providing collaborative learning experience using high-fidelity simulation as a teaching modality for interdisciplinary students/interprofessional education ${ }^{[13,34.35]}$.

This study offered unique insights to professional interactions involving four pre-professional students groups and many members of the healthcare team from a health science center. Most interprofessional studies use two student groups, mainly medicine and nursing ${ }^{[10,16,36-38]}$, physical and occupational therapy ${ }^{[35]}$, or surgery residents, faculty surgeons, and emergency department nurses ${ }^{[13]}$. A recently published article used five healthcare professions including student groups and licensed professionals in an obstetrics simulated emergency: nurse practitioner, physician assistant, undergraduate nursing students, medical students, and obstetrics residents ${ }^{[39]}$. Although different assessment instruments were used, significant improvements were reported in team collaboration scores ${ }^{[10,13,38,39]}$, attitudes and knowledge ${ }^{[16,36,37]}$, communication ${ }^{[37,39]}$, and recognition of quality team skills in video vignettes ${ }^{[36]}$. Our study also reported improvement in teamwork and communication scores.

Limitations of the study were the small sample size and loss of participants between fall and spring. Scheduling conflicts were the main reason for attrition. Small sample sizes were reported by other researchers ${ }^{[10,38,39]}$ and highlighted the difficulty in scheduling between the different professional schools, which was a challenge in our study. Worzala and colleagues ${ }^{[38]}$ reported attrition between pretest and posttest, losing half of students for posttest, while another study reported $21 \%$ of subjects not completing post-tests ${ }^{[39]}$.

Larger sample sizes were achieved with all day team training ${ }^{[16,35,36]}$ and involvement of students from two universities ${ }^{[16]}$. Our study used a total of seven faculty for observation, performance rating, and debriefing, whereas Robertson and colleagues ${ }^{[36]}$ used 40 facilitators for team training and Posmontier and colleagues ${ }^{\text {[39] }}$ trained 20 faculty. Faculty and university resources are important factors to consider when planning interprofessional education with or without HPS.

While the scenario used for this study did not identify the "patient's” ethnicity or culture, it was implied as Caucasian based on skin color and verbalizations. A richer more diverse learning experience might occur if moulage were used to change the skin color along with the use of varied speech patterns. Scenarios of this type would prepare the interprofessional team for realistic practice settings. A brief review of literature did not reveal any studies on interprofessional high-fidelity simulations assessing cultural competency.

Interprofessional HFSBT was effective in an ICU setting with students from four professional programs. Findings support repeat training in a six month period. The need for distributive training was reported in a systematic review of effective learning with high-fidelity medical simulations ${ }^{[40]}$. Thirty-nine percent $(n=43)$ of the articles in the review identified repetitive practice as a basic feature of high-fidelity simulations ${ }^{[40]}$. Common challenges were reported in single interprofessional simulations and in this distributed training. Thus, the question is how to overcome the challenges to incorporate best practices for HFSBT distributed over time to all interprofessional student groups. 


\section{Conclusion}

Future research is needed on the best interval between training to ensure skill retention and the best structure for training to ensure translation of knowledge to the bedside. Methodology issues would be a challenge; for example, how can a researcher demonstrate that knowledge is actually used and outcomes are the result of HPSBT instead of other variables. Also, what is the impact of culture on outcomes of HPSBT and translation of skills to the bedside; management of various patient cultures or interaction with different discipline cultures offer rich learning scenarios. Use of cultural competence in a crisis situation would make an interesting study that could contribute to a gap in the literature in this area. This type of scenario would be an excellent learning experience to determine if behaviors translate to patient care environments where patients are of different backgrounds.

HFSBT has the potential to break down the cultural and practice barriers between professions. This study brought together four different groups of pre-licensure professional students. The next steps are to examine if these teamwork competencies are evident in the work environment. How can this training make a change in the culture of an ICU and result in a better working environment and ultimately improved patient care. This is educations charge from national organizations, but it is really our duty as healthcare professionals and educators.

\section{References}

[1] Mazzocco K, Petitti DB, FongKT, Bonacum D, Brookey J, Graham S, Lasky RE, Sexton JB, Thomas EJ. Surgical team behaviors and patient outcomes. American Journal of Surgery. 2009; 197(5): 678-685. PMid:18789425 http://dx.doi.org/10.1016/j.amjsurg.2008.03.002

[2] Mishra A, Catchpole K, Dale T, McCulloch P. The influence of non-technical performance on technical outcome in laparoscopic cholecystectomy. Surgical Endoscopy. 2008; 22(1): 68-73. PMid:17479324 http://dx.doi.org/10.1007/s00464-007-9346-1

[3] Baker DP, Gustafson S, Beaubien J, Salas E, Barach P. Medical Teamwork and Patient Safety The Evidence-Based Relation. AHRQ Publication No. 05-0053, Chapter 4 Medical Team Training [Internet]. 2005. Available from: http://www.ahrq.gov/qual/medteam/ (31 May 2012 date last accessed).

[4] Greiner AC, Knebel E. Health Professions Education: A Bridge to Quality. Institute of Medicine. Washington D.C.: National Academies Press, 2003.

[5] Angelini D Interdisciplinary and Interprofessional Education: What are the Key Issues and Considerations for the Future? The Journal of Perinatal \& Neonatal Nursing. 2011; 25(2): 175-179. PMid:21540696

[6] Reese CE, Jeffries PR, Engum SA. Learning together: using simulations to develop nursing and medical student collaboration. Nursing Education Perspectives. 2010; 31(1): 33-37. PMid:20397478

[7] Bleakley A, Boyden J, Hobbs A, Walsh L, Allard J. Improving teamwork climate in operating theatres: the shift from multiprofessionalism to interprofessionalism. Journal of Interprofessional Care. 2006; 20(5): 461-470. PMid:17000472 http://dx.doi.org/10.1080/13561820600921915

[8] Cooke M, Irb DM, O’Brien BC. Summary of educating physicians: A call for reform of medical school and residency. Carnegie Foundation for the Advancement of Teaching [Internet]. 2010. Available from: http://www.carnegiefoundation.org/elibrary/summary-educating-physicians (30 November 2011 date last accessed).

[9] Kohn LT, Corrigan J, Donaldson MS (Eds.). To Err is Human: Building a Safer Health System. Institute of Medicine. Washington D.C.: National Academies Press. 2000.

[10] Dillon P, Noble K, Kaplan L. Simulation as a Means to Foster Collaborative Interdisciplinary Education. Nursing Education Research. 2009; 30(2): 87-90.

[11] Leape L, Berwick D, Clancy C, Conway J, Gluck P, Guest J, et al. Transforming healthcare: A safety imperative.” 2009; 18: 424-428. PMid:19955451 http://dx.doi.org/10.1136/qshc.2009.036954

[12] Beaubien JM, Baker DP. The use of simulation for training teamwork skills in health care: how low can you go? Quality \& Safety in Health Care. 2004; 13: 51-56. http://dx.doi.org/10.1136/qshc.2004.009845

[13] Capella J, Smith S, Philp A, Putnam T, Gilbert C, Fry W, et al. Teamwork Training Improves the Clinical Care of Trauma Patients. Journal of Surgical Education. 2010; 67(6): 439-444. PMid:21156305 http://dx.doi.org/10.1016/j.jsurg.2010.06.006 
[14] Siassakos, D, Draycott TJ, Crofts JF, Hunt LP, Winter C, Fox R. More to teamwork than knowledge, skill and attitude. British Journal of Obstetrics and Gynecology. 2010; 117: 1262-1269. PMid:20618313 http://dx.doi.org/10.1111/j.1471-0528.2010.02654.x

[15] Siassakos, D, Bristowe K, Draycott TJ, Angouri J, Hambly H, Winter C, et al. Clinical efficiency in a simulated emergency and relationship to team behaviors: A multisite cross-sectional study. British Journal of Obstetrics and Gynecology. 2011; 118: 596-607. PMid:21291509 http://dx.doi.org/10.1111/j.1471-0528.2010.02843.x

[16] Hobgood C, Sherwood G, Frush K, Hollar D, Maynard L, Foster B, et al. Teamwork training with nursing and medical students: does the method matter? Results of an interinstitutional, interdisciplinary collaboration. Quality and Safety in Health Care. 2010; 19(6): e25. Epub 2010 Apr 27. PMid:20427311 http://dx.doi.org/10.1136/qshc.2008.031732

[17] Wilson K.A, Burke CS, Priest HA, Salas E. Promoting health safety through training high reliability teams. Quality \& Safety in Health Care. 2005; 14: 303-309. PMid:16076797 http://dx.doi.org/10.1136/qshc.2004.010090

[18] PaigeJT, Kozmenko V, Yang T, Gururaja RP, Cohn I, Hilton C, et al. The mobile mock operating room: bringing team training to the point of care. In Henriksen K., J.B. Battles, M.A. Keyes, and M.L. Grady, (Eds.).Advances in Patient Safety: New Directions and Alternative Approaches (Vol. 3: Performance and Tools). Rockville, MD: Agency for Healthcare Research and Quality. 2008.

[19] Paige J, Kozmenko V, Morgan B, Howell DS, Chauvin S, Hilton C, et al. From the flight deck to the operating room: an initial pilot study of the feasibility and potential impact of true interdisciplinary team training using high-fidelity simulation.” 2007; 64(6): 369-77. PMid:18063272 http://dx.doi.org/10.1016/j.jsurg.2007.03.009

[20] Paige JT, Kozmenko V, Yang T, Gururaja PR, Hilton CW, Cohn I Jr., et al. High-fidelity, simulation-based, interdisciplinary operating room team training at the point of care. Surgery. 2009; 145(2): 138-146. PMid:19167968 http://dx.doi.org/10.1016/j.surg.2008.09.010

[21] Paige JT, Kozmenko V, Yang T, Gururaja RP, Hilton CW, Cohn I Jr., et al. Attitudinal changes resulting from repetitive training of operating room personnel using of high-fidelity simulation at the point of care. American Surgeon. 2009; 75(7): 584-90; discussion 590-591. PMid:19655602

[22] Gururaja RP, Yang T, Paige JT, Chauvin SW. Examining the effectiveness of debriefing at the point of care in simulation-based operating room team training. In Henriksen, K., J.B. Battles, M.A. Keyes, and M.L. Grady, (Eds.). Advances in Patient Safety: New Directions and Alternative Approaches (Vol. 3: Performance and Tools). Rockville, MD: Agency for Healthcare Research and Quality. 2008.

[23] Kozmenko V, Paige J, Chauvin S. Initial implementation of mixed reality simulation targeting teamwork and patient safety. Student Health Technology Informatics. 2008; 132: 216-221. PMid:18391289

[24] Pearson M, Smith D. Debriefing in experience-based learning. In Boud, D., R. Keogh, and D. Walker. (Eds.). Reflection: Turning Experience into Learning. 1985; 69-85.

[25] Fanning RM, Gaba DM. The role of debriefing in simulation-based learning. Simulation in Healthcare. 2007 ; 2: $115-125$. PMid:19088616 http://dx.doi.org/10.1097/SIH.0b013e3180315539

[26] Thiagarajan S, Thiagarajan R, England J. Six phases of debriefing [Internet]. 2004. Available from: http://www.thiagi.com/pfp/IE4H/february2004.html\#Debriefing (30 November 2011, date last accessed).

[27] Fernandez R, Vozenilek JA, Hegarty CB, Motola I, Reznek M, Phrampus PE, et al. Developing expert medical teams: toward an evidence-based approach. Academic Emergency Medicine. 2008; 15: 1025-1036. PMid:18785937 http://dx.doi.org/10.1111/j.1553-2712.2008.00232.x

[28] Chauvin SW, Paige JT, Yang T. Operating Room Teamwork Assessment Scales (ORTAS). Office of Medical Education Research and Services, School of Medicine, Louisiana State University Health Sciences Center, New Orleans 2006. Revised July 2007, 2008.

[29] PaigeJT, Aaron DL, Yang T, Howell DS, Hilton CW, Cohn I Jr., et al. Implementation of a preoperative briefing protocol improves accuracy of teamwork assessment in the operating room. American Surgeon. 2008; 74(9): 817-823. PMid:18807669

[30] Paige JT, Aaron DL, Yang T, Howell DS, Chauvin SW. Improved operating room teamwork via SAFETY Prep: a rural community hospital’s experience. World Journal of Surgery. 2009; 33(6): 1181-1187. PMid:19350322 http://dx.doi.org/10.1007/s00268-009-9952-2

[31] Frankel A, Gardner R, Maynard L, Kelly A. Using the communication and teamwork skills (CATS) assessment to measure health care team performance. The Joint Commission Journal on Quality and Patient Safety. 2007; 33(9): 549-558. PMid:17915529

[32] Malec JF, Torsher LC, Dunn WF, Wiegmann DA, Arnold J, Brown DA, et al. The Mayo high performance teamwork scale: Reliability and validity for evaluating key crew resource management skills. Simulation in Healthcare: The Journal of the Society for Simulation in Healthcare. 2007; 2(1): 4-10. PMid:19088602 http://dx.doi.org/10.1097/SIH.0b013e31802b68ee

[33] Geis GL, Pio B, Pendergrass TL, Moyer MR, Patterson MD. Simulation to assess the safety of new healthcare teams and new facilities. Simulation in Healthcare. 2011; 6: 125-133. PMid:21383646 http://dx.doi.org/10.1097/SIH.0b013e31820dff30 
[34] Garrett B, MacPhee M, Jackson C. High-fidelity patient simulation: Considerations for effective learning. Nursing Education Perspectives. 2010; 31(5): 309-313. PMid:21086870

[35] Shoemaker MJ, Beasley J, Cooper M, Perkins R, Smith J, Swank C. A method for providing high-volume interprofessional simulation encounters in physical and occupational therapy education programs. Journal of Allied Health. 2011; 40(1): e15-21. PMid:21399842

[36] Robertson B, Kaplan B, Atallah H, Higgins M, Lewitt MJ, Ander DS. The use of simulation and a modified TeamSTEPPS curriculum for medical and nursing student team training. Simulation in Healthcare. 2010; 5(6): 332-337. PMid:21330818 http://dx.doi.org/10.1097/SIH.0b013e3181f008ad

[37] Stewart M, Kennedy N, Cuene-Grandidier H. Undergraduate interprofessional education using high-fidelity paediatric simulation. The Clinical Teacher. 2010; (7): 90-96. PMid:21134155 http://dx.doi.org/10.1111/j.1743-498X.2010.00351.x

[38] Worzala KT, Glaser KM, McGinley A. A collaborative curriculum for medical and nursing students. Medical Education. 2006; 40(5): 478. PMid:16635151 http://dx.doi.org/10.1111/j.1365-2929.2006.02431.x

[39] Issenberg SB, McGaghie WC, Petrusa ER, Gordon DL, Scalese RJ. Features and uses of high-fidelity medical simulations that lead to effective learning: a BEME systematic review. Medical Teacher. 2005; 27(1): 10-28. PMid:16147767

http://dx.doi.org/10.1080/01421590500046924 